\title{
An Improved Macro Model of Traffic Flow with the Consideration of Ramps and Numerical Tests
}

\author{
Zhongke Shi, Wenhuan Ai, and Dawei Liu \\ College of Automation, Northwestern Polytechnical University, No. 127 Youyi Road (West), Beilin, Xian, Shaanxi 710072, China \\ Correspondence should be addressed to Zhongke Shi; shizknwpu@126.com
}

Received 8 April 2015; Revised 11 June 2015; Accepted 14 June 2015

Academic Editor: Xiaosong $\mathrm{Hu}$

Copyright (C) 2015 Zhongke Shi et al. This is an open access article distributed under the Creative Commons Attribution License, which permits unrestricted use, distribution, and reproduction in any medium, provided the original work is properly cited.

\begin{abstract}
We present an improved macro model for traffic flow based on the existing models. The equilibrium point equation of the model is obtained. The stop-and-go traffic phenomenon is described in phase plane and the relationship between traffic jams and system instability is clearly shown in the phase plane diagrams. Using the improved model, some traffic phenomena on a highway with ramps are found in this paper. The numerical simulation is carried out to investigate various nonlinear traffic phenomena with a single ramp generated by different initial densities and vehicle generation rates. According to the actual road sections of Xi'an-Baoji highways, the situations of morning peak with several ramps are also analyzed. All these results are consistent with real traffic, which shows that the improved model is reasonable.
\end{abstract}

\section{Introduction}

In recent years, traffic jams has become more and more serious. They do not only cause a large number of costs but they also have a negative impact on the environment and energy sustainability. Therefore, researchers have made many efforts to develop transportation electrification to alleviate the impact. Hu et al. [1,2] analyzed the energy efficiencies of a series plug-in hybrid electric bus with different energy management strategies and battery sizes and comparatively examined three different electrochemical energy storage systems for a hybrid bus powertrain. Recently, they [3] also investigated the optimal component sizing and power management of a fuel cell/battery hybrid bus. Sun et al. [4] presented a traffic data-enabled predictive energy management framework for plug-in hybrid electric vehicles. Furthermore, they [5] studied the velocity predictors for predictive energy management in hybrid electric vehicles. However, many other physicists and engineers have tried to develop traffic models with the aim of optimizing traffic flow. During the past decades, lots of traffic models have been constructed to replicate the formation mechanism and inherent law of the traffic phenomena. In microscopic view, the traffic flow system was regarded as a complex self-driven manyparticle system composed of a large number of vehicles. The microscopic traffic flow models investigated the dynamical behavior of a single vehicle and the interactions between the vehicles, so they can also describe the traffic phenomena of the whole system. Among them, the car-following model is a favorable type of traffic models describing the driver's following behavior in view of the stimulus from its preceding vehicle. On the macroscopic view, physicists paid close attention on the collective behavior of traffic. Due to the analogy of vehicle stream with gas stream or fluid stream, large numbers of the gas kinetic models or fluid-dynamic models have been developed to approximately describe the traffic phenomena. Based on these models, researchers can use a lot of system simulation methods to analyze the traffic phenomena.

The traffic flow on a highway with the ramp has been studied for decades through observation and modeling. Lee et al. [6] studied the presence of the external vehicle flux through ramps and found a new kind of traffic phenomenon, called "recurring humps" (RH). In this state, the density and the flow oscillated periodically and the oscillations concentrated around the ramp. Gupta and Katiyar [7] studied the phase transition on a highway in a modified anisotropic continuum model with an on-ramp. Huang [8] observed an interesting phase: jam-max.-free when the on-ramp is placed before the off-ramp. He also demonstrated that the bulk properties on the roadway are totally controlled by the ramp flow through 
the boundaries. Tang et al. [9] indicated that ramps often have different effects on the main road traffic during the morning rush period and the evening rush period and that the effects are related to the initial status of the main road traffic flow.

However, these models cannot completely describe the various complex phenomena resulted by different input and output conditions on ramps. In particular, the phenomena of fixed vehicle generation rate but increasing initial homogeneous density with a single ramp, the situation of morning peak, and the congested traffic stream with several ramps are rarely studied in the past. In this paper, we present an improved macro model for traffic flow to analyze these phenomena on a highway with a single ramp and multiple ramps. Moreover, we introduce a completely different method to describe traffic phenomena in the phase plane diagrams from a stability perspective. The variable substitution is adopted in the models and the traffic congestion corresponds to the unstable system in phase plane. So the traffic flow problems can be converted into the system stability problems.

The remainder of the paper is organized as follows. In Section 2, we present an improved continuum model based on the existing traffic flow model. In Section 3, we deduce the equilibrium point equation of the model. In Section 4, we analyze the well-known stop-and-go waves using the phase plane diagrams based on the improved model and compare them with the traditional temporal evolution of vehicle density. In Section 5, we use the improved model to describe various nonlinear phenomena on a highway with a single ramp. In Section 6, the traffic flow on a highway with multiple ramps is also studied and the actual traffic phenomena of Xi'an-Baoji highways are discussed. We conclude the paper in Section 7.

\section{Models}

The macroscopic traffic flow models consider vehicles as interacting particles and consider traffic flow as a onedimensional compressible flow of these particles. The study of macroscopic traffic flow models began with the LWR model proposed by Lighthill and Whitham [10] and Richards [11]. To overcome the shortage of the LWR model, Payne [12] developed a higher order model by using a dynamic equation for the mean velocity. Hereafter, many researchers presented a great number of models based on Payne's model [13-15]. However, these models fail to describe the property that the characteristic speeds are always less than or equal to the macroscopic flow speed. Later, Zhang [16] proposed a macroscopic traffic flow model which overcomes the backward travel problem. Gupta and Katiyar [17] also developed an anisotropic continuum model which is referred to as GK model. Although these models can describe many complex traffic phenomena, they cannot be used to directly explore the effects of ramps since they do not consider this factor. So far, some theoretical models have been developed to study the effects of ramps [6-9]. However, these models cannot completely describe the various complex phenomena resulted by different input and output conditions on ramps. In particular, the phenomena of fixed vehicle generation rate but increasing initial homogeneous density with a single ramp, the situation of morning peak, and the congested traffic stream with several ramps are rarely studied in the past. In this paper, we present an improved macro model for traffic flow on a highway with ramps based on the GK model as follows:

$$
\begin{aligned}
\frac{\partial \rho}{\partial t}+\frac{\partial(\rho v)}{\partial x}= & s(x, t) \\
\frac{\partial v}{\partial t}+v \frac{\partial v}{\partial x}= & \frac{1}{\tau} \cdot\left[V_{e}(\rho)-v\right]+\frac{1}{\tau} \\
& \cdot V_{e}^{\prime}(\rho)\left[\frac{1}{2 \rho} \frac{\partial \rho}{\partial x}+\frac{1}{6 \rho^{2}} \frac{\partial^{2} \rho}{\partial x^{2}}-\frac{1}{2 \rho^{3}}\left(\frac{\partial \rho}{\partial x}\right)^{2}\right] \\
& -2 \beta c(\rho) \frac{\partial v}{\partial x}
\end{aligned}
$$

where $\rho$ is the density; $v$ is the velocity; $x$ and $t$ represent space and time, respectively; $\tau$ is the driver's reaction time; $\beta$ is a nonnegative dimensionless parameter; $V_{e}[\rho(x, t)]$ is the optimal velocity function and has the following form [18]:

$$
\begin{aligned}
& V_{e}[\rho] \\
& \quad=v_{f}\left\{\left[1+\exp \left(\frac{\rho / \rho_{m}-0.25}{0.06}\right)\right]^{-1}-3.72 \times 10^{-6}\right\} .
\end{aligned}
$$

Consider $V_{e}^{\prime}(\rho)=d V_{e}(\rho) / d \rho, v_{f}$ is the free-flow speed, $\rho_{m}$ is the maximum or jam density, and $c(\rho) \prec 0$ is the traffic sound speed given by

$$
c^{2}(\rho)=-\frac{a V_{e}^{\prime}(\rho)}{2} .
$$

Consider $s(x, t)$ is the flow generation rate. For simplicity, we here adopt the definition of flow generation rate in Jiang et al. [19]; that is

$$
s(x, t)= \begin{cases}\frac{q_{\mathrm{ramp}}}{L_{\mathrm{ramp}}} & \forall x \in \Omega_{\mathrm{ramp}}, \\ 0 & \text { else, }\end{cases}
$$

where $\Omega_{\text {ramp }}$ is the region of the ramp, $L_{\text {ramp }}$ is the length of the ramp, and $q_{\text {ramp }}$ is the total ramp flow. We here define $q_{\text {ramp }}$ as follows:

$$
q_{\mathrm{ramp}}=q_{\mathrm{ramp}}^{\mathrm{on}}-q_{\mathrm{ramp}}^{\mathrm{off}}
$$

where $q_{\text {ramp }}^{\text {on }}$ is the input flow of the on-ramp and $q_{\text {ramp }}^{\text {off }}$ is the output flow of the off-ramp.

Furthermore, we employ a simple transformation as follows:

$$
\begin{aligned}
\sigma & =\frac{1}{v} \\
\eta & =\frac{1}{\rho_{m}-\rho} .
\end{aligned}
$$

Substituting the variables into (1), we have a new traffic flow model as follows:

$$
\frac{\partial \eta}{\partial t}=-\frac{1}{\sigma} \frac{\partial \eta}{\partial x}+\frac{\rho_{m} \eta^{2}-\eta}{\sigma^{2}} \frac{\partial \sigma}{\partial x}+\eta^{2} \cdot s(x, t)
$$




$$
\begin{aligned}
\frac{\partial \sigma}{\partial t} & =\left(2 \beta \sqrt{-\frac{v_{e}^{\prime}(\eta)}{2 \tau}}-\frac{1}{\sigma}\right) \frac{\partial \sigma}{\partial x}-\frac{\sigma^{2} v_{e}^{\prime}(\eta)}{\tau} \\
& \cdot\left\{\frac{1}{2 \eta\left(\rho_{m} \eta-1\right)} \cdot \frac{\partial \eta}{\partial x}+\frac{1}{6\left(\rho_{m} \eta-1\right)^{2}} \cdot \frac{\partial^{2} \eta}{\partial x^{2}}\right. \\
& \left.-\left(\frac{1}{3 \eta\left(\rho_{m} \eta-1\right)^{2}}+\frac{1}{2 \eta\left(\rho_{m} \eta-1\right)^{3}}\right)\left(\frac{\partial \eta}{\partial x}\right)^{2}\right\} \\
& -\frac{\sigma^{2} v_{e}(\eta)}{\tau}+\frac{\sigma}{\tau} .
\end{aligned}
$$

Similarly, substituting the variables into (2), the equilibrium velocity $v_{e}(\eta)$ is as follows:

$$
\begin{aligned}
& V_{e}(\eta) \\
& \quad=v_{f}\left\{\left[1+\exp \left(\frac{0.75-1 / \eta \rho_{m}}{0.06}\right)\right]^{-1}-3.72 \times 10^{-6}\right\} .
\end{aligned}
$$

According to the variable substitution $\sigma=1 / v$, we can see that as long as the traffic becomes congested and the vehicles velocity goes to zero, the state variable $\sigma$ will approach infinity. Likewise, from the variable substitution, $\eta=1 / \rho_{m}-\rho$, we can see that if the vehicle density becomes saturated, the state variable $\eta$ will approach infinity in the same way. So we can use the phase plane diagrams about the variable $\eta$ or $\sigma$ to describe clearly the relationship between traffic jams and system instability. As long as the traffic has a very small density fluctuation, the value of $\eta$ and $\sigma$ will change sharply. Moreover, as long as there is traffic jam formation, the value of $\eta$ and $\sigma$ will approach infinity. The more the value of $\eta$ and $\sigma$ increases, the greater the fluctuation of the vehicle density is and the more unstable the traffic system is. On the contrary, the system becomes more stable, so the problem of traffic flow could be converted into that of system stability. We can describe all kinds of nonlinear traffic phenomena with the phase plane diagrams and determine whether there will be traffic congestion or other abnormal phenomena from a global stability point. It may be possible to apply some mathematical tools such as branch and bound to the nonlinear stability analysis of traffic system. We can find the equilibrium solutions and some bifurcations of the new model to regulate the stability of traffic system in the future work.

\section{The Equilibrium Point Equation Analysis}

When the traffic system reaches equilibrium state, the density and velocity of the whole road will not change with time. Moreover, when the traffic system reaches some special equilibrium points, the density and velocity of the whole road will not change with time and displacement at the same time. In order to find these equilibrium solutions of the new model, the equilibrium point equation of the system is analyzed firstly.
When system (7) does not change with time, we have

$$
\begin{aligned}
& \frac{\partial \eta}{\partial t}=0 \\
& \frac{\partial \sigma}{\partial t}=0 .
\end{aligned}
$$

It is assumed that the input flow of the on-ramp is equal to the output flow of the off-ramp. So we have $s(x, t)=0$. By substituting (9) into (7), the equilibrium points satisfy the formula as follows:

$$
\begin{aligned}
& \left(\eta-\rho_{m} \eta^{2}\right) \frac{\partial \sigma}{\partial x}+\sigma \frac{\partial \eta}{\partial x}=0, \\
& \left(\frac{1}{\sigma}-2 \beta \sqrt{\left.-\frac{v_{e}^{\prime}(\eta)}{2 \tau}\right)}\right) \frac{\partial \sigma}{\partial x}+\frac{\sigma^{2} v_{e}^{\prime}(\eta)}{\tau}\left\{\frac{1}{2 \eta\left(\rho_{m} \eta-1\right)}\right. \\
& \quad \cdot \frac{\partial \eta}{\partial x}+\frac{1}{6\left(\rho_{m} \eta-1\right)^{2}} \cdot \frac{\partial^{2} \eta}{\partial x^{2}} \\
& \left.-\left(\frac{1}{3 \eta\left(\rho_{m} \eta-1\right)^{2}}+\frac{1}{2 \eta\left(\rho_{m} \eta-1\right)^{3}}\right)\left(\frac{\partial \eta}{\partial x}\right)^{2}\right\} \\
& \quad+\frac{\sigma^{2} v_{e}(\eta)}{\tau}-\frac{\sigma}{\tau}=0 .
\end{aligned}
$$

Equation (10a) can be rewritten as

$$
\frac{1}{\sigma} \frac{\partial \sigma}{\partial x}=\frac{1}{\eta\left(\rho_{m} \eta-1\right)} \frac{\partial \eta}{\partial x}
$$

The right hand side of (11) can be written as

$$
\begin{aligned}
\frac{1}{\eta\left(\rho_{m} \eta-1\right)} \frac{\partial \eta}{\partial x} & =\frac{\partial f(\eta)}{\partial \eta} \frac{\partial \eta}{\partial x}=\left(\frac{a}{\eta}+\frac{b}{\rho_{m} \eta-1}\right) \frac{\partial \eta}{\partial x} \\
& =\left(\frac{\rho_{m}}{\rho_{m} \eta-1}-\frac{1}{\eta}\right) \frac{\partial \eta}{\partial x}
\end{aligned}
$$

So,

$$
\begin{aligned}
f(\eta) & =\ln \left(\rho_{m} \eta-1\right)-\ln \eta=\ln \left(\frac{\rho_{m} \eta-1}{\eta}\right) \\
& =\ln \left(\rho_{m}-\frac{1}{\eta}\right) .
\end{aligned}
$$

By integrating (11) at both ends, we have

$$
\ln \sigma=f(\eta)+\text { const }=\ln k_{0}\left(\rho_{m}-\frac{1}{\eta}\right) .
$$

$k_{0}$ is a nonzero constant and (14) can be rewritten as

$$
\sigma=k_{0}\left(\rho_{m}-\frac{1}{\eta}\right) \text {. }
$$


By substituting (15) and (11) into (10b), we obtain

$$
\begin{gathered}
\frac{k_{0}^{2} \eta V_{e}^{\prime}(\eta)\left(\rho_{m} \eta-1\right)}{6 \tau} \frac{\partial^{2} \eta}{\partial x^{2}}+\frac{k_{0}^{2} V_{e}^{\prime}(\eta)}{\tau}\left[\frac{1}{3}\left(1-\rho_{m} \eta\right)-\frac{1}{2}\right] \\
\cdot\left(\frac{\partial \eta}{\partial x}\right)^{2}+\left[\eta^{2}-2 \beta k_{0} \eta\left(\rho_{m} \eta-1\right) \sqrt{-\frac{V_{e}^{\prime}(\eta)}{2 \tau}}\right. \\
\left.+\frac{k_{0}^{2} V_{e}^{\prime}(\eta)\left(\rho_{m} \eta-1\right)^{2}}{2 \tau}\right] \frac{\partial \eta}{\partial x} \\
+\frac{k_{0} \eta\left(\rho_{m} \eta-1\right)^{2}}{\tau}\left[k_{0}\left(\rho_{m} \eta-1\right) V_{e}(\eta)-\eta\right]=0 .
\end{gathered}
$$

In summary, the system equilibrium points satisfy the following equations:

$$
\begin{aligned}
& \sigma=k_{0}\left(\rho_{m}-\frac{1}{\eta}\right) \\
& \frac{k_{0}^{2} \eta V_{e}^{\prime}(\eta)\left(\rho_{m} \eta-1\right)}{6 \tau} \frac{\partial^{2} \eta}{\partial x^{2}}+\frac{k_{0}^{2} V_{e}^{\prime}(\eta)}{\tau}\left[\frac{1}{3}\left(1-\rho_{m} \eta\right)-\frac{1}{2}\right] \\
& \cdot\left(\frac{\partial \eta}{\partial x}\right)^{2}+\left[\eta^{2}-2 \beta k_{0} \eta\left(\rho_{m} \eta-1\right) \sqrt{-\frac{V_{e}^{\prime}(\eta)}{2 \tau}}\right. \\
& \left.+\frac{k_{0}^{2} V_{e}^{\prime}(\eta)\left(\rho_{m} \eta-1\right)^{2}}{2 \tau}\right] \frac{\partial \eta}{\partial x} \\
& +\frac{k_{0} \eta\left(\rho_{m} \eta-1\right)^{2}}{\tau}\left[k_{0}\left(\rho_{m} \eta-1\right) V_{e}(\eta)-\eta\right]=0 \\
& V_{e}(\eta)=v_{f}\left\{\left[1+\exp \left(12.5-\frac{1}{0.06 \rho_{m} \eta}\right)\right]^{-1}-3.72\right. \\
& \left.\times 10^{-6}\right\} \cdot
\end{aligned}
$$

The meaning of every parameter is the same as above. If the initial values of $\eta$ and $\sigma$ are given by the solution of (17), the density and velocity of the whole road will not change with time. At the same time, we can see from (15) that the product of density and velocity is equal to $1 / k_{0}$.

Furthermore, when system (17) also does not change with displacement, we obtain the equilibrium points equation as follows:

$$
\begin{aligned}
& \sigma=k_{0}\left(\rho_{m}-\frac{1}{\eta}\right) \\
& \frac{k_{0} \eta\left(\rho_{m} \eta-1\right)^{2}}{\tau}\left[k_{0}\left(\rho_{m} \eta-1\right) V_{e}(\eta)-\eta\right]=0 .
\end{aligned}
$$

Next, we analyze the solution of (18b). $\eta$ cannot be zero according to $\eta=1 /\left(\rho_{m}-\rho\right)$. If $\rho_{m} \eta-1=0$, then $\rho$ is equal to 0 . In this case, $\eta$ is trivial equilibrium point and has no practical significance. So we only need to investigate the following equation:

$$
k_{0}\left(\rho_{m} \eta-1\right) V_{e}(\eta)-\eta=0
$$

Equation (19) can be written as

$$
V_{e}(\eta)=\frac{\eta}{k_{0}\left(\rho_{m} \eta-1\right)}
$$

At the same time, (18a) can be written as $\sigma=k_{0}\left(\rho_{m} \eta-\right.$ $1) / \eta$. So we can see from (20) that $V_{e}(\eta)=1 / \sigma=v$.

Therefore, we may conclude that if the value of initial density is set as a random constant in the reasonable range of traffic flow and the initial velocity is given as the equilibrium velocity which is corresponding to the initial density, the density and velocity of the whole road will not change with time and displacement. These conclusions are also consistent with the phenomena observed in realistic traffic flow.

\section{The Stop-and-Go Traffic Phenomena on the Phase Plane}

The stop-and-go traffic phenomena are international wellknown nonlinear phenomena. Traditional researches on it mainly focused on using the figures of temporal development of density through the original traffic flow models. The new model mentioned above can also describe it through the phase plane diagrams from a system stability perspective. The comparisons and discussions between the two methods by numerical experiments were given as follows. Here we assume that the input flow of the on-ramp is equal to the output flow of the off-ramp.

The stop-and-go phenomena can be observed in the amplification of a small disturbance. In this section, we simulate the stop-and-go phenomena with respect to an amplified localized perturbation in an initial homogeneous condition. The following initial variation of the average density $\rho_{0}$ is used as in [20]:

$$
\begin{aligned}
& \rho(x, 0)=\rho_{0}+\Delta \rho_{0}\left\{\cosh ^{-2}\left[\frac{160}{L}\left(x-\frac{5 L}{16}\right)\right]\right. \\
& \left.-\frac{1}{4} \cosh ^{-2}\left[\frac{40}{L}\left(x-\frac{11 L}{32}\right)\right]\right\} \quad x \in[0, L] \\
& v(x, 0)=V(\rho(x, 0)) \quad x \in[0, L],
\end{aligned}
$$

where $\rho_{0}$ is the initial vehicle density, $\Delta \rho_{0}=0.01 \mathrm{veh} / \mathrm{m}$ is the amplitude of localized perturbation, and $L=32.2 \mathrm{~km}$ is the length of road section under consideration. The dynamic approximate boundary condition was given by

$$
\begin{aligned}
& \rho(1, t)=\rho(2, t), \\
& \rho(L, t)=\rho(L-1, t), \\
& v(1, t)=v(2, t), \\
& v(L, t)=v(L-1, t) .
\end{aligned}
$$

For computational purpose, the space domain was divided into equal intervals of length of $100 \mathrm{~m}$ and time 
interval was chosen as $1 \mathrm{~s}$. The related parameters of our model were as follows:

$$
\begin{aligned}
\beta & =2, \\
\tau & =14 \mathrm{~s}, \\
v_{f} & =30 \mathrm{~m} / \mathrm{s} \\
\rho_{m} & =0.2 \mathrm{veh} / \mathrm{m}, \\
\rho_{0} & =0.052 \mathrm{veh} / \mathrm{m}, \\
\Delta \rho_{0} & =0.01 \mathrm{veh} / \mathrm{m} .
\end{aligned}
$$

The critical density values of the GK model corresponding to the parameters above were $0.037 \mathrm{veh} / \mathrm{m}$ and $0.091 \mathrm{veh} / \mathrm{m}$, which can easily be found out by the stability condition [17]. The traffic flow will be unstable between these critical densities. The small disturbance in these initial homogeneous conditions will be amplified, and the stop-andgo phenomena will occur.

Traditionally, people used the temporal evolution of vehicle density or velocity to describe the stop-and-go waves, such as in [17, 21-24]. In particular, [17] employed the GK model to analyze it. The variation range of vehicle density is $0-0.25 \mathrm{veh} / \mathrm{m}$ and velocity is $0-30 \mathrm{~m} / \mathrm{s}$. So there are rather limited changes in the diagrams about these variables. When the traffic becomes congested, the vehicle density and velocity both tend to a specific value. We cannot see significant changes from the traditional temporal development of density or velocity. However, through our variable substitutions, the state variable $\eta$ and $\sigma$ both tend to infinity. As long as the traffic has a small fluctuation, the value of $\eta$ or $\sigma$ will change sharply. Moreover, as long as there is traffic jam formation, the value of $\eta$ or $\sigma$ will approach infinity. Using the new model by such variable substitution, we can describe clearly the relationship between traffic jams and system instability in the phase plane. The numerical solution of $\eta$ and $\sigma$ can be obtained by applying the finite difference method on the new model. Then we analyze the stop-and-go phenomena with four phase plane diagrams. The coordinate systems of them are $(\eta, \partial \eta / \partial t),(\eta, \partial \eta / \partial x),(\sigma, \partial \sigma / \partial t)$, and $(\sigma, \partial \sigma / \partial x)$, respectively. Through the four graphs the variation of density or velocity with time or sections can be investigated more clearly. Thus we can completely convert the fluctuations of traffic flow into the stability analysis charts.

Figure 1 shows the unstable traffic situation with small perturbations divergence when the initial density was set to $0.052 \mathrm{veh} / \mathrm{m}$. Figure $1(\mathrm{a})$ is the temporal evolution of vehicle density. Since the value of initial density we set was in the unstable range, the amplitude of the initial small perturbations grows in time, leading to traffic instability. A complex localized structure consisting of two or more clusters forms. This situation corresponds to stop-and-go traffic.

A cross-sectional analysis was made along a time axis of Figure 1(a) to observe the variation of density with time when the section is fixed. Since the state variable $\eta$ increases strictly monotonously with the density, we draw the change curves of $\eta$ with time on each road section in Figure 1(b). Similarly, since $\sigma$ is inversely proportional to the vehicle velocity, we draw the change curves of $\sigma$ with time on each road section in Figure 1(d). If the traffic approaches congestion, the density will approach the jam density and the velocity will be close to zero. The state variables $\eta$ and $\sigma$ will tend to infinity and the system will become unstable.

If the curves in Figures 1(b) and 1(d) are drawn one by one in the order of road section, we can find the curve of each downstream section moved toward the outer ring of upstream section. It shows that density fluctuations were gradually amplified toward the upstream section and the average velocity gradually reduced. The initial small perturbations spread upstream and were divergent, leading to the whole traffic system instability.

The phase plane diagrams emphasized the instable situation of the traffic system we are chiefly concerned about. It can be seen that the value of $\eta$ and $\sigma$ will change sharply in Figures $1(b)$ and $1(d)$ even when there is a very small fluctuation in Figure 1(a). When the vehicle density approached congestion, the value of $\eta$ and $\sigma$ will get larger. The corresponding curves of such traffic jams accounted for a large proportion in the graphs, while most small amplitude density fluctuations accounted for a quite small percentage and they are just centered in a small area near the initial value.

Then we made a cross-sectional analysis along a displacement axis of Figure 1(a) to observe the variation of density with displacement when time is fixed. Figures 1(c) and 1(e) are, respectively, the change curves of $\eta$ and $\sigma$ per second on the whole road. If these curves are drawn one by one in the order of time, we can see all the curves change with time from the inner circle to the outer ring and many of them tend to infinity. It also demonstrates that density fluctuations of the whole road are gradually amplified with time. The amplitude of the initial small perturbations grows in time. The whole traffic system is unstable. Compared with the temporal evolution of density, the phase plane diagrams can more clearly reflect the density variation of current time and the next time. So through the phase plane diagrams we can convert directly the traffic jam phenomenon into the curves of instability system. The result is more obvious when the traffic system is more instable.

Figure 2 is the change curves of density and $\eta$ on the ninetieth road section which changes along with time. It can be seen from Figure 2(a) that there were many density fluctuations at this road section in the first ten minutes and this situation corresponds to stop-and-go traffic.

It reflects the density fluctuations outstandingly in Figure 2(b). We find out the starting point of the curve to observe its trajectory. It moves from the innermost point which is labeled as 6.6225 to the outermost circle and then turns toward the small circles inside. A circle of the phase plane diagram corresponds to a density fluctuation of the density curve chart. The variation of the circle ring in horizontal direction corresponds to the amplitude of a density fluctuation and in vertical direction it corresponds to the change rate of a density fluctuation. So the phase plane diagrams clearly reflect the size and speed of the density fluctuations. These results are consistent with the stopand-go traffic phenomena described by the density curve 


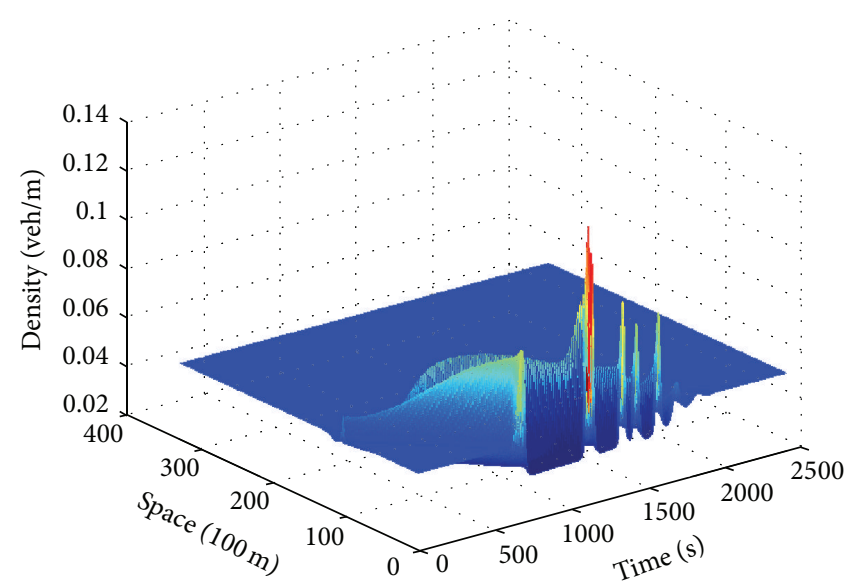

(a)

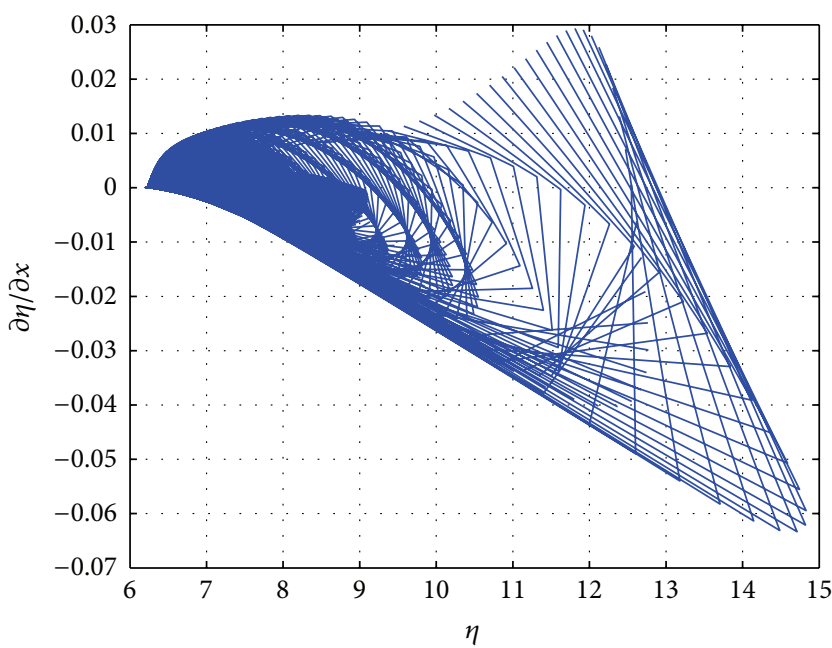

(c)

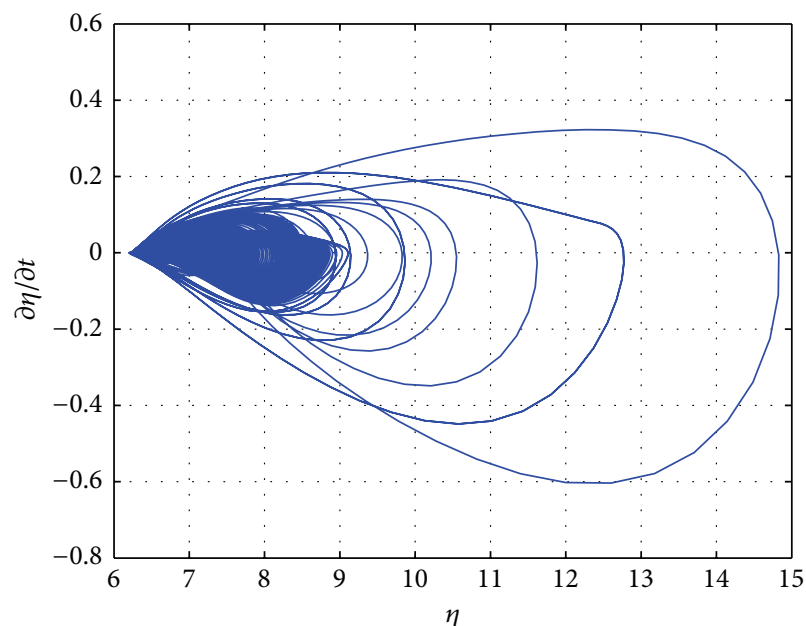

(b)

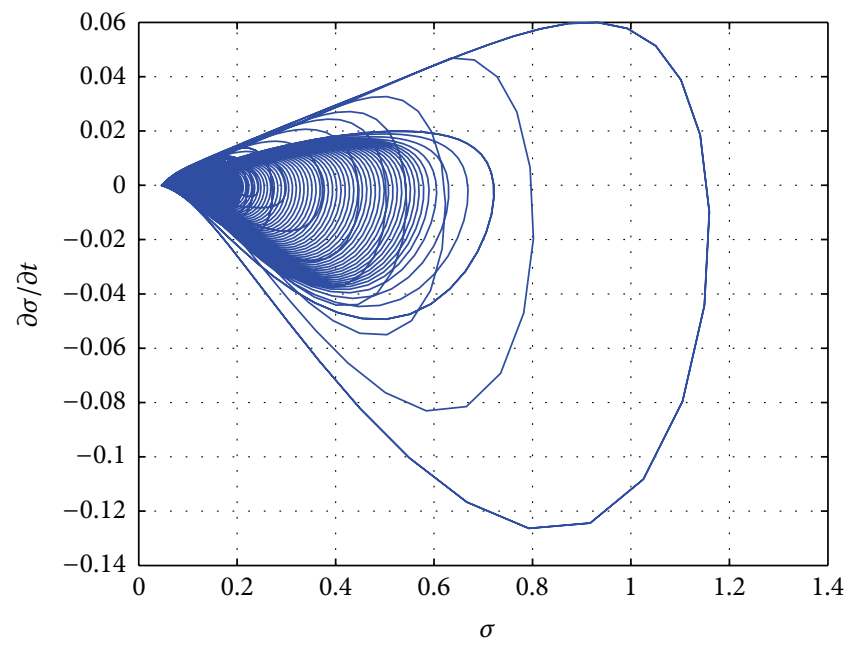

(d)

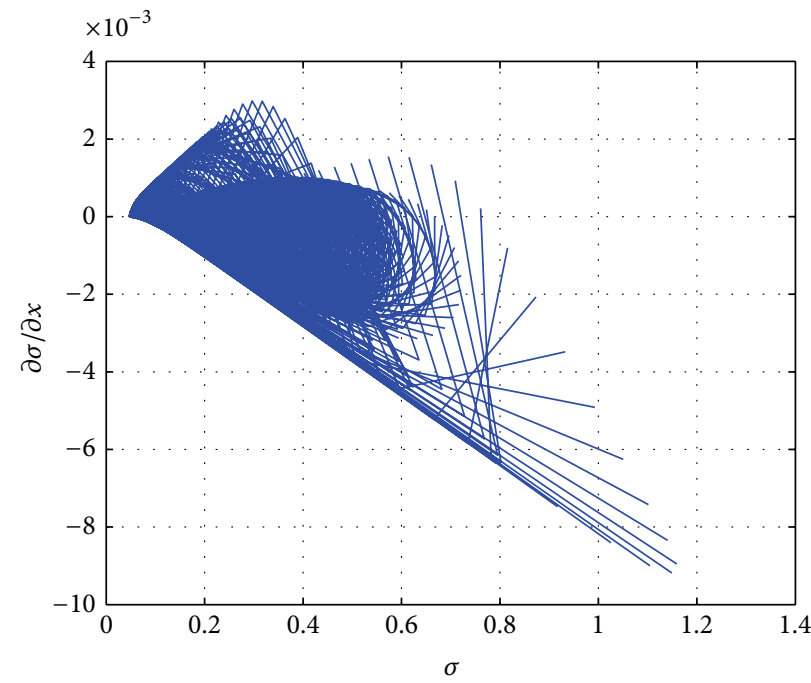

(e)

FIGURE 1: Evolution of small perturbation under the initial density $0.052 \mathrm{veh} / \mathrm{m}$. (a) The temporal evolution of vehicle density. (b) The phase plane diagram of $(\eta, \partial \eta / \partial t)$. (c) The phase plane diagram of $(\eta, \partial \eta / \partial x)$. (d) The phase plane diagram of $(\sigma, \partial \sigma / \partial t)$. (e) The phase plane diagram of $(\sigma, \partial \sigma / \partial x)$. 


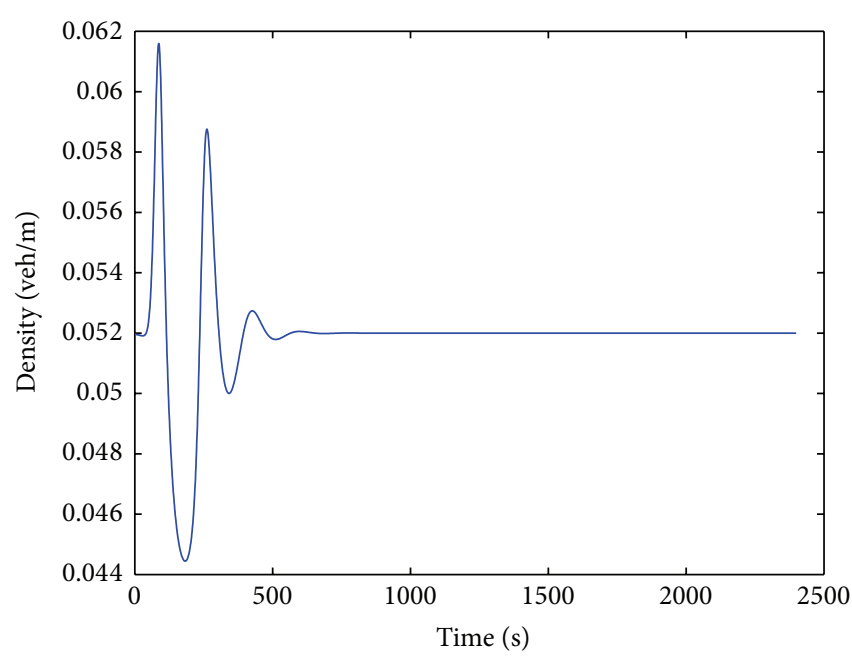

(a)

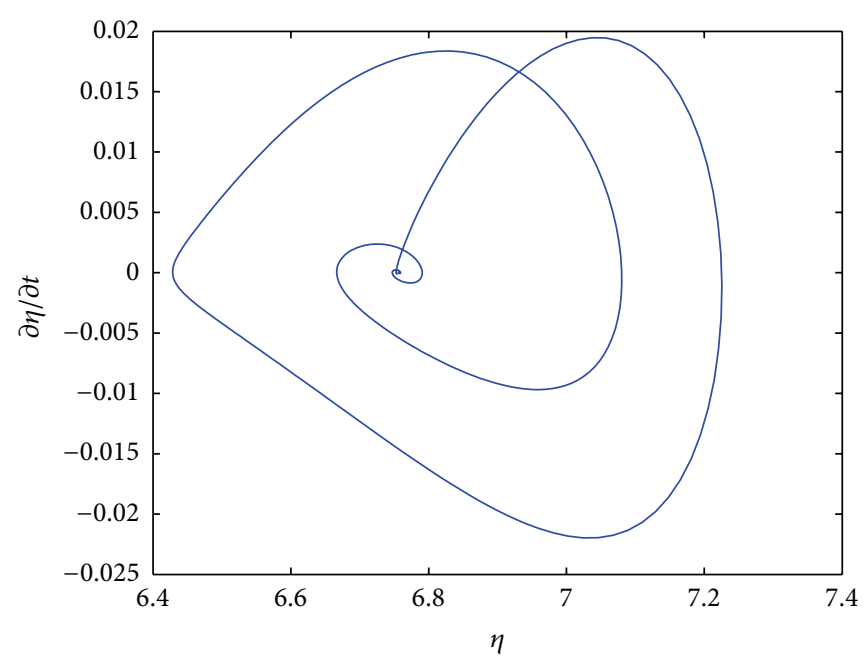

(b)

Figure 2: (a) The density-time variation curve on the ninetieth road section. (b) The phase plane diagram of $(\eta$, $\partial \eta / \partial t)$ on the ninetieth road section.

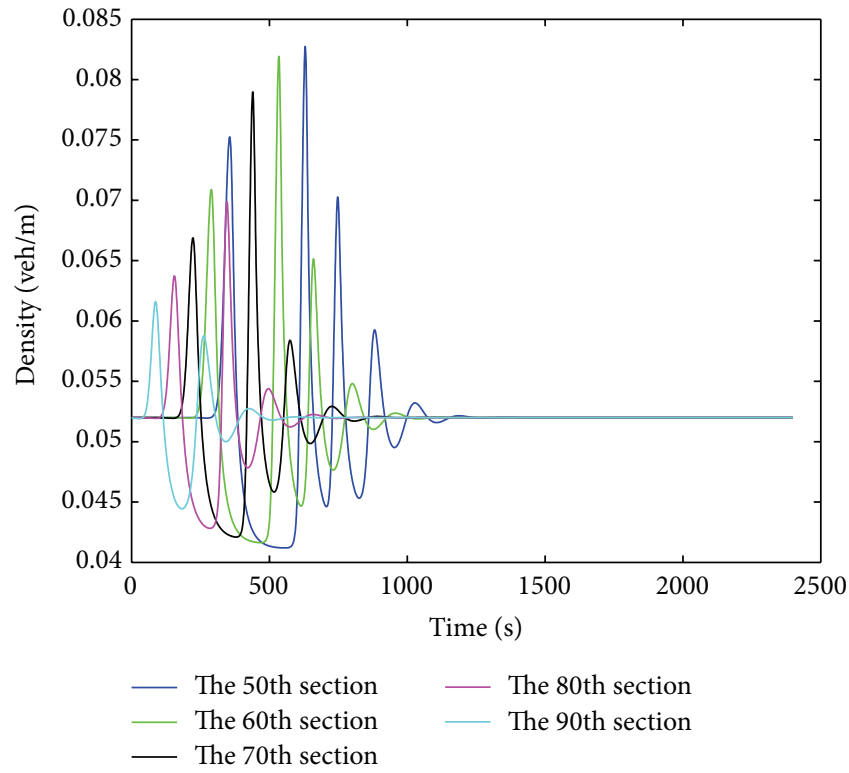

(a)

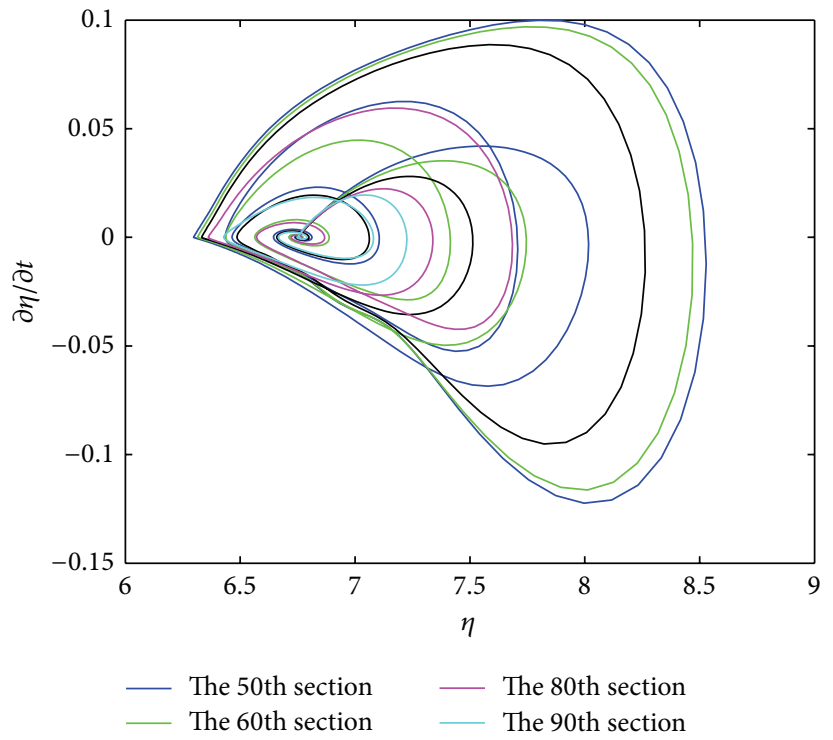

(b)

FIGURE 3: (a) Density-time variation curves on the ninetieth, the eightieth, the seventieth, the sixtieth, and the fiftieth road sections. (b) The phase plane diagram of $(\eta, \partial \eta / \partial t)$ on the ninetieth, the eightieth, the seventieth, the sixtieth, and the fiftieth road sections.

chart. Moreover, the phase plane diagrams mainly reflect the unstable traffic conditions we are chiefly concerned with.

Figure 3 is the change curves of density and $\eta$ in the first 40 minutes on the ninetieth, the eightieth, the seventieth, the sixtieth, and the fiftieth road sections which change along with time. As you can see from Figure 3(a), the amplitude of the density fluctuation on the 90th section is small, as shown by the cyan line. It is growing toward the upper section of the road because the initial small perturbations spread upstream and are divergent. In Figure 3(b), the variation of $\eta$ on the 90th section is small, as shown by the cyan circle inside. It grows toward the upper sections and reaches the largest on the 50th section as shown by the blue circle outside. So it also shows the initial perturbation is amplified upstream. Although there are very small density fluctuations in Figure 3(a), the variation range of $\eta$ is big in Figure 3(b). The phase plane diagram mainly reflects the density fluctuations.

Figure $4(a)$ is the density variation curves of the 1100th, the 1101st, the 1102nd, the 1103rd, the 1104th, the 1105th, and the 1106th seconds of the whole road section. The curves almost coincide together and we cannot see the 

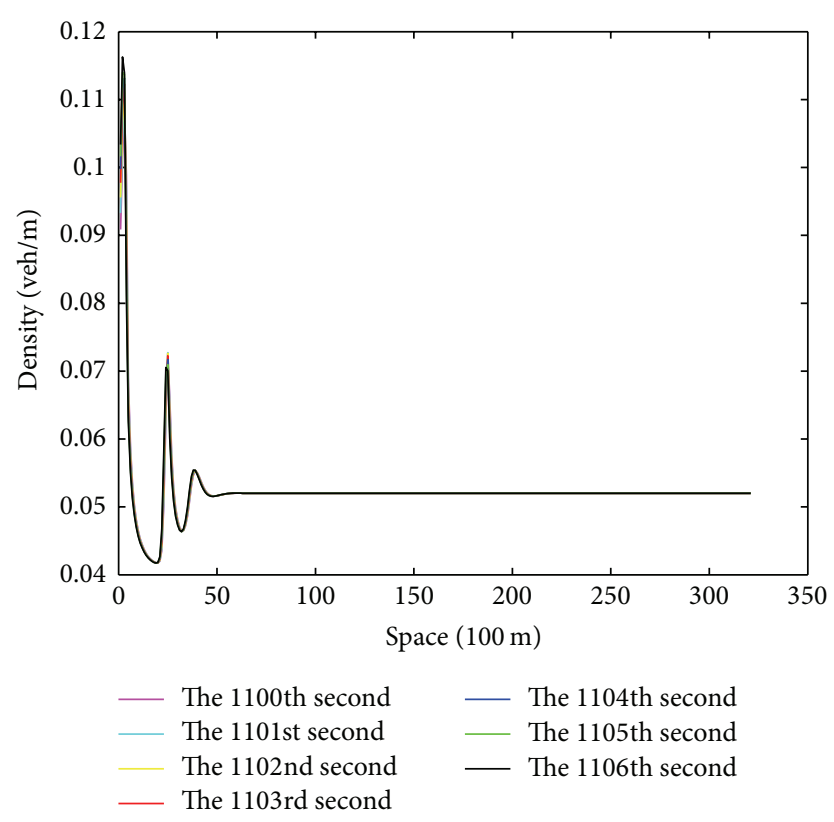

(a)

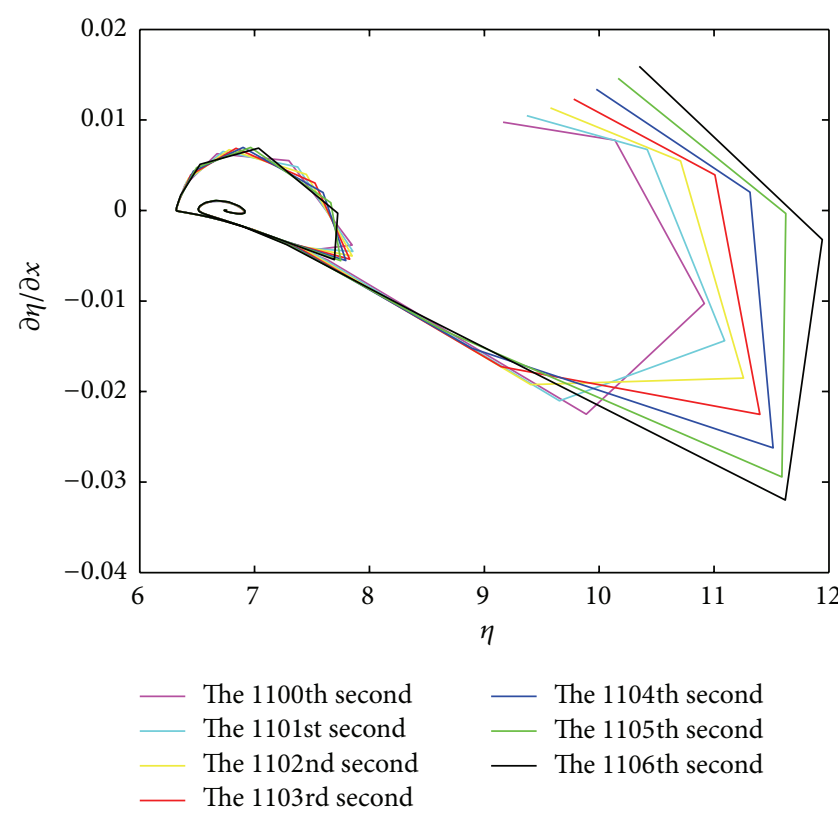

(b)

Figure 4: (a) Density-space variation curves on the 1100th, the 1101st, the 1102nd, the 1103rd, the 1104th, the 1105th, and the 1106th seconds of the whole road section. (b) The phase plane diagrams of $(\eta, \partial \eta / \partial x)$ on the 1100th, the 1101st, the 1102nd, the 1103rd, the 1104th, the 1105th, and the 1106th seconds of the whole road section.

obvious changes. Figure $4(\mathrm{~b})$ is the phase plane diagrams corresponding to these times and it can clearly show that the curves enlarge per second from inside to outside; that means the amplitude of the density on the whole road grows in time and the traffic system is divergent.

Figure 5(a) is the velocity-space variation curves of the 679 th, 680th, the 681st, the 682nd, the 683rd, the 684th, and the 685th seconds on the whole road section. All of the curves almost coincide together and we cannot see the obvious changes. However, the corresponding phase plane diagram in Figure 5(b) obviously shows that the inner circle of the current time turns to the outer ring of the next second and all of the curves tend to infinity. So it clearly reflects unstable traffic phenomena.

Comparing the phase plane diagrams with the temporal evolution of density shows that the stop-and-go traffic phenomena described by the new model are consistent with that described by the original model. But, unlike the previous temporal evolution of density, the phase plane diagram focuses on the density fluctuation we mainly care for and does not highlight the most homogeneous state of stable traffic flow. Moreover, it describes the variation of density or velocity with time or sections more clearly.

\section{The Traffic Phenomena on a Highway with a Single Ramp}

Although some empirical studies have been conducted to evaluate traffic data on highways with ramps, they cannot completely describe the various complex phenomena of fixed vehicle generation rate but increasing initial homogeneous density with a single ramp and the situation of morning peak. As it is very difficult to investigate the traffic phenomena induced by ramps, we use simulations by our model to describe the effects that ramps have on a main road. We take the test road section as $32.2 \mathrm{~km}$ long and set a ramp in the middle of the road section. We assume that the number of vehicles through an on-ramp is 36 veh more than that through an off-ramp every meter per hour. The length of the ramp is $100 \mathrm{~m}$. So the fixed vehicle generation rate is $0.0001 \mathrm{veh} / \mathrm{m} / \mathrm{s}$. Other parameter values are the same as in Section 4. The results are shown in Figure 6.

It is clear from Figure 6(a) that since the vehicle generation rate is a small constant and the initial density of the main road is also lower than the down-critical unstable density, the vehicles coming from the on-ramp can drive quickly downstream and will not have any effect on the upstream traffic. That is to say, vehicles upstream can keep their speeds even when they drive past the ramp. So the density increments just appear from the on-ramp and reduce downstream gradually. The ramp has a small effect on the main road at this situation and this phenomenon will seldom appear because the main road density is relatively high.

In Figure 6(b), since the initial density is just above the down-critical unstable density, the ramp can disturb the stability of the main road traffic. A small quantity of vehicles coming from the ramp can be seen as a small localized perturbation on the initial homogeneous traffic flow. The amplitude of perturbation grows in time and eventually forms the stop-and-go traffic. So the ramp produces stop-and-go traffic when the main road density is between two critical values. The fluctuation amplitude of traffic flow is much larger 


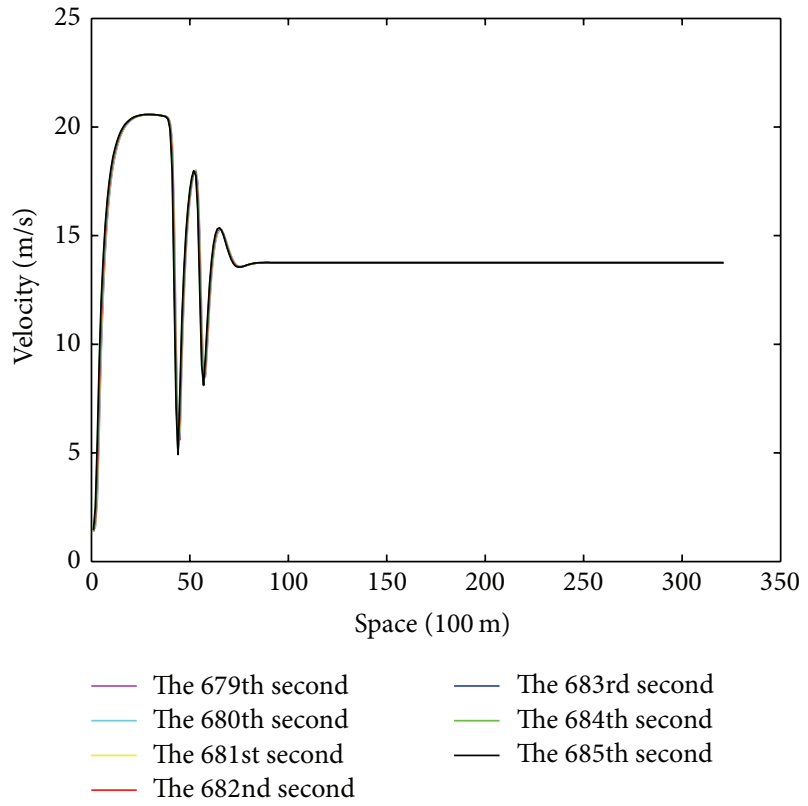

(a)

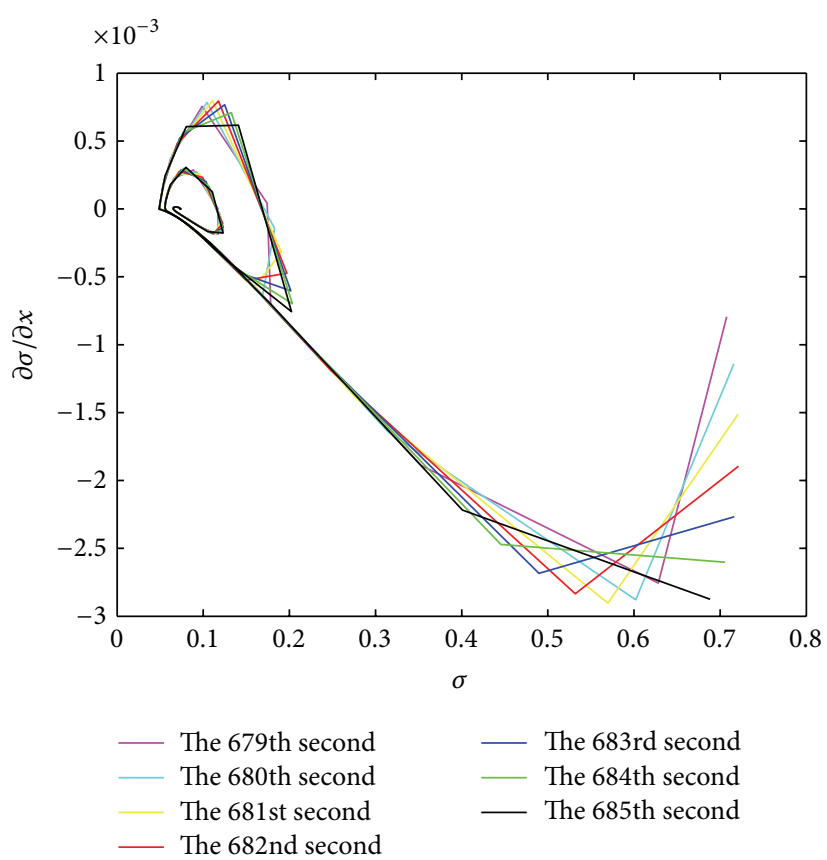

(b)

Figure 5: (a) Velocity-space variation curves on the 679th, the 680th, the 681st, the 682nd, the 683rd, the 684th, and the 685th seconds of the whole road section. (b) The phase plane diagrams of $(\sigma, \partial \sigma / \partial x)$ on the 679 th, the $680 \mathrm{th}$, the 681st, the 682nd, the 683rd, the 684 th, and the 685th seconds of the whole road section.

than the vehicle generation rate on ramp. These phenomena often appear during the rush hours. In this case, the inflow of the ramp should be reduced to improve the main road traffic.

Figure 6(c) shows that when the initial density becomes greater than the up-critical density, a stable regime of the model is reached again and the perturbation is dissipated. Most of the cars which entered from the ramp accumulate on the ramp road section and the ramp becomes jammed very quickly. It shows the vehicles queue near the on-ramp when the main road density is relatively high. The ramp should be closed at this situation.

The phenomena described above are the situation of fixed vehicle generation rate but increasing initial homogeneous density. Next, we will analyze the phenomena of fixed initial homogeneous density but changing vehicle generation rate. The morning peak is a common phenomenon in traffic flow. In order to simulate it approximately, we set the value of vehicle generation rate on the ramp to change with a sine wave and the maximal amplitude of it is $36 \mathrm{veh} / \mathrm{m} / \mathrm{h}$. The number of vehicles which entered the ramp is increasing at the beginning of the peak hours in the morning and again is decreasing gradually after the peak hours. The initial uniform density is set to $0.027 \mathrm{veh} / \mathrm{m}$ and other parameter values are the same as above. The temporal evolution of vehicle density is shown in Figure 7.

It is clear from Figure 7 that the density is increased first and gradually decreased with the morning peak on the ramp section which we set in the middle of the road. The density increment of the ramp section spreads gradually downstream. As the initial density of the main road is very low and the vehicle generation rate is also small, the vehicles coming from the on-ramp can drive quickly downstream.

\section{The Traffic Phenomena on a Highway with Multiple Ramps}

This section primarily analyzes traffic phenomena on a highway with multiple ramps by using the real traffic data. According to the new model and phase plane diagrams, several situations of morning traffic in Xian-Baoji Highway (China) were simulated. The total length in Xian to Baoji freeway is 187.404 kilometers and the station distances of all road sections are shown in Table 1.

There are ramps on each toll station according to the practical distribution. To simulate the morning traffic, we can assume the values of vehicle generation rate on every ramp all change with a sine wave and the maximal amplitude of them are all $0.001 \mathrm{veh} / \mathrm{m} / \mathrm{s}$. Firstly, we set the initial density of the whole road at $0.025 \mathrm{veh} / \mathrm{m}$ which is in the stable range of our model. For computational purpose, the space domain was divided into equal intervals of length of $200 \mathrm{~m}$ and the time interval was chosen as $1 \mathrm{~s}$. Other parameter values used were as follows:

$$
\begin{aligned}
\beta & =6, \\
v_{f} & =125 \mathrm{~m} / \mathrm{s}, \\
\tau & =14 \mathrm{~s}, \\
\rho_{m} & =0.25 \mathrm{veh} / \mathrm{m} .
\end{aligned}
$$




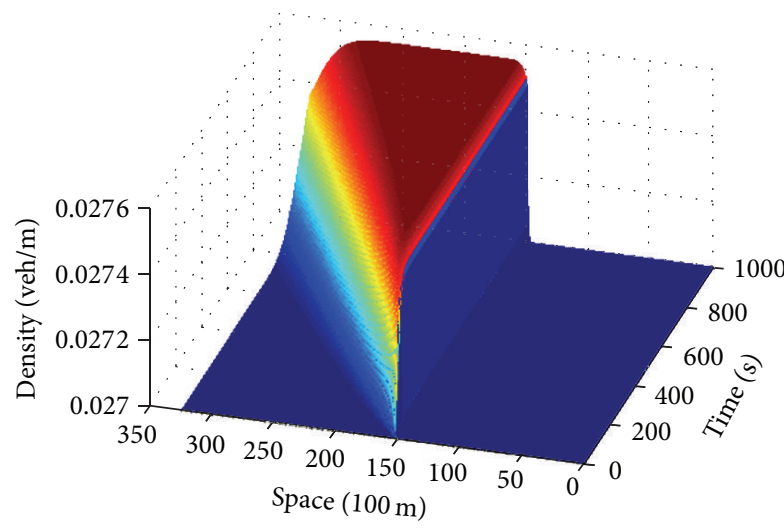

(a) $\rho_{0}=0.027 \mathrm{veh} / \mathrm{m}$

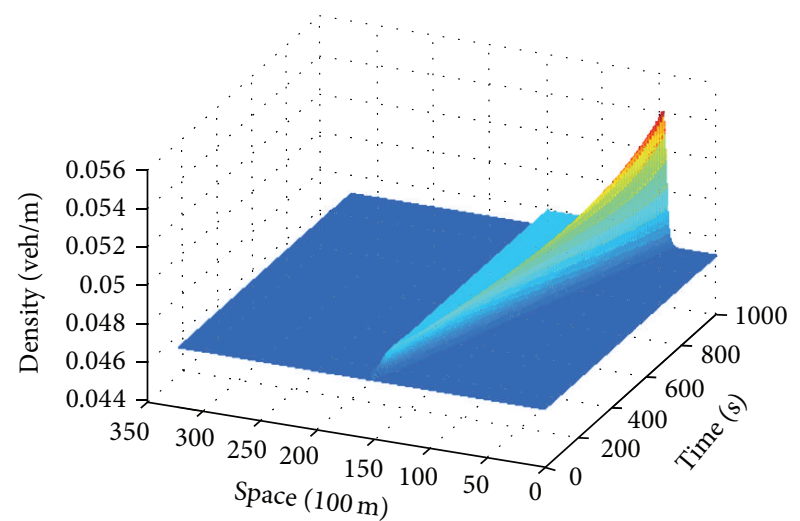

(b) $\rho_{0}=0.047 \mathrm{veh} / \mathrm{m}$

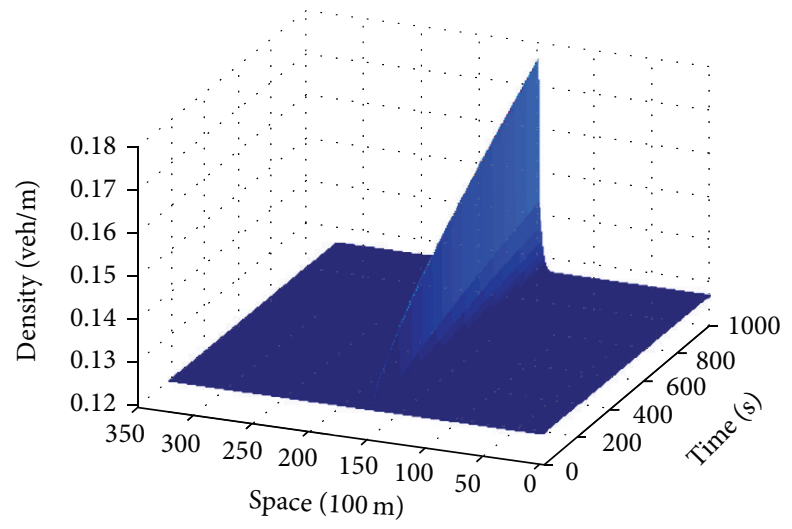

(c) $\rho_{0}=0.127 \mathrm{veh} / \mathrm{m}$

FIgURE 6: The density temporal evolution with initial homogeneous traffic and fixed vehicle generation rate.

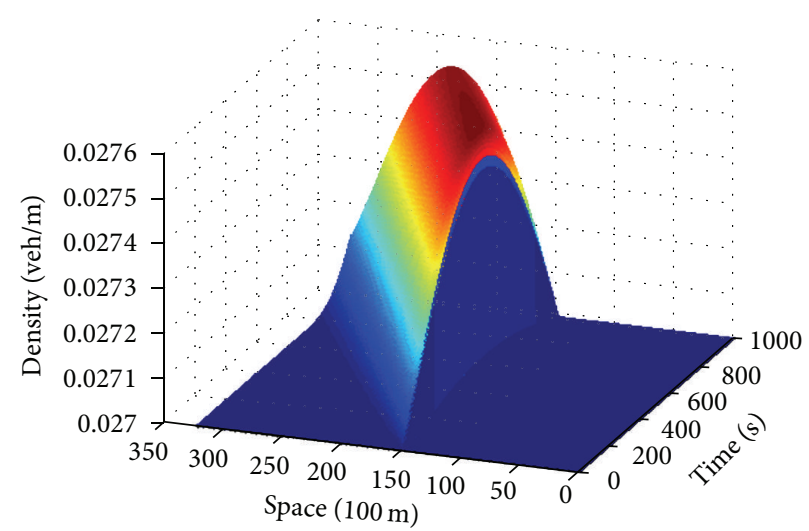

FIGURE 7: The density temporal evolution with initial homogeneous traffic of amplitude $\rho_{0}=0.027 \mathrm{veh} / \mathrm{m}$ to simulate the morning peak.

TABLE 1: The general situation of Xian-Baoji highway.

\begin{tabular}{|c|c|c|c|c|c|c|c|c|c|c|c|c|}
\hline $\begin{array}{l}\text { Toll stations or } \\
\text { control points }\end{array}$ & Sanqiao & Xianyang & $\begin{array}{c}\text { Xian } \\
\text { Yang } \\
\mathrm{Xi}\end{array}$ & Xingping & Wugong & Yangling & $\begin{array}{l}\text { Jiang } \\
\text { Zhang }\end{array}$ & Changxing & Meixian & $\begin{array}{c}\text { Cai Jia } \\
\text { Po }\end{array}$ & Guozhen & Baoji \\
\hline $\begin{array}{l}\text { The station } \\
\text { distances (KM) }\end{array}$ & $\begin{array}{c}\text { Starting } \\
\text { point }\end{array}$ & 9.698 & 6.092 & 13.594 & 27.419 & 11.83 & 13.08 & 10.515 & 11.725 & 9.855 & 21.855 & 17.815 \\
\hline
\end{tabular}




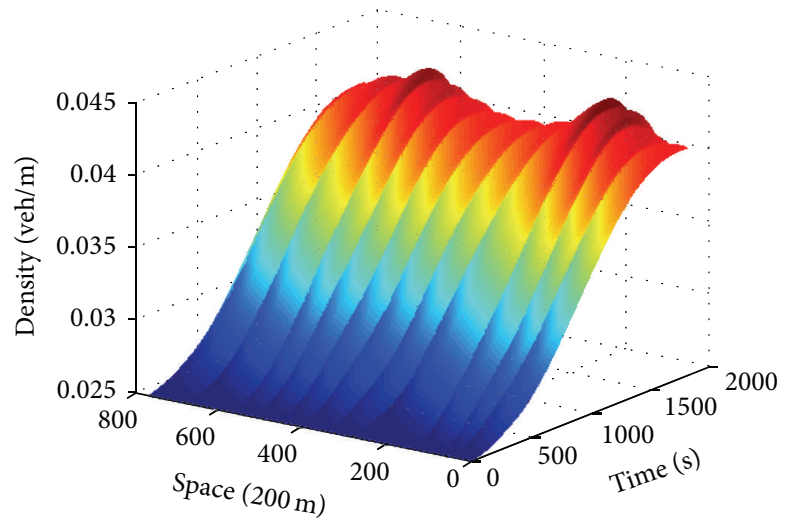

FIGURE 8: The density temporal evolution on the Xi'an-Baoji highway with initial homogeneous traffic of amplitude $\rho_{0}=0.025 \mathrm{veh} / \mathrm{m}$.

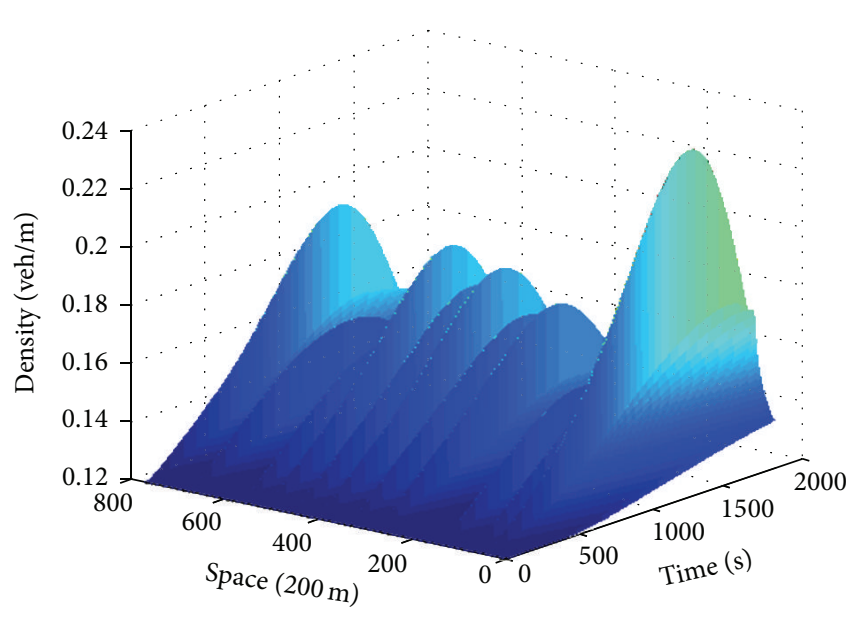

(a)

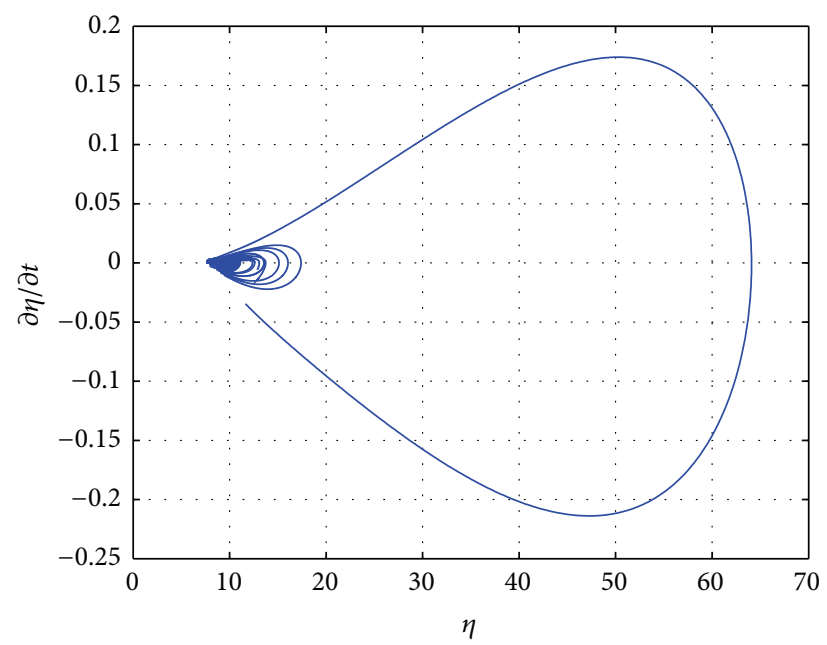

(b)

Figure 9: The density temporal evolution and phase plane diagrams on the Xian-Baoji highway with initial homogeneous traffic of amplitude $\rho_{0}=0.12 \mathrm{veh} / \mathrm{m}$. (a) The temporal evolution of vehicle density. (b) The phase plane diagram of $(\eta, \partial \eta / \partial t)$.

The meanings of these parameters are the same as in Section 2. The result illustrated by the temporal evolution of density was shown in Figure 8.

Since we assumed that the number of vehicles which entered the ramp was always greater than that exited the ramp at rush hours, the values of vehicle generation rate on every ramp were positive and this could eventually cause the density of the whole road to increase continuously. However, the densities were far less than the jam density and the traffic did not reach the congestion state because of the small initial homogeneous density.

To further simulate the morning traffic approaching the value of jam density on the Xian-Baoji highway, we increased the initial density of $0.12 \mathrm{veh} / \mathrm{m}$ and remained the value of vehicle generation rate; the temporal evolution of vehicle density and phase plane diagrams can be compared in Figure 9.

In Figure 9(a), since the initial density is increased and the vehicles continually enter from the ramp, the density of the whole road increases greatly and some road sections approach the congestion eventually. Figure 9(b) is the combination of variation curves of $\eta$ on each road section during the first 30 minutes. Since the number of the vehicles which entered the ramp increases first and then decreases before and after the morning peak, the trajectory of $\eta$ also increases first and then decreases. So there are lots of irregular cycles in the figure corresponding to different density fluctuations. When the amplitude value of the density fluctuation is small, the cycle radius is small. However, the radius of cycle becomes very large when the density reaches the jam density. Although such cycles are not many, they account for very big proportion in the graph.

If we continually increased the initial density of $0.127 \mathrm{veh} / \mathrm{m}$ and other conditions were the same as above, the density of the whole road will increase continually with time and reach the jam density eventually. When the sum of the traffic demand of the upstream section and the traffic flow expected into highway is greater than the traffic capacity of the downstream section, ramp metering can be applied usually to restrict the number of the vehicles which entered 


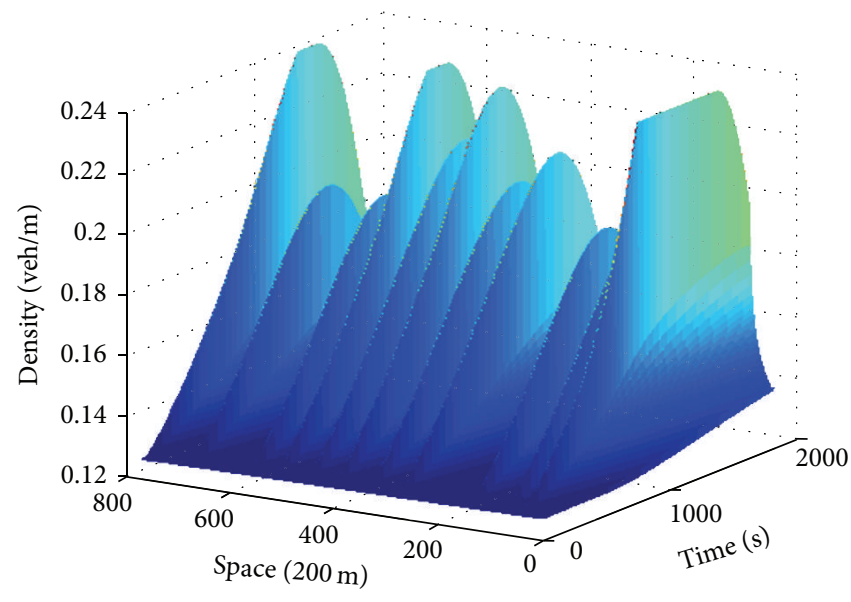

(a)

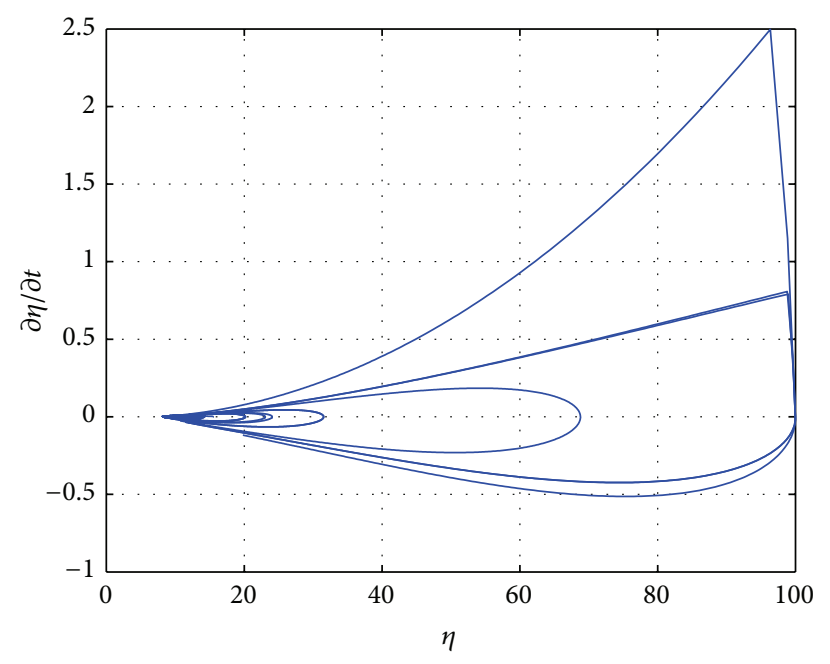

(b)

FIGURE 10: The density temporal evolution and phase plane diagrams on the Xi'an-Baoji highway with initial homogeneous traffic of amplitude $\rho_{0}=0.127 \mathrm{veh} / \mathrm{m}$. (a) The temporal evolution of vehicle density. (b) The phase plane diagram of $(\eta, \partial \eta / \partial t)$.

the ramp and excess vehicles will wait in the queue. So we let $\rho=\rho_{m}-10^{-2}$ when $\rho \geq \rho_{m}-10^{-2}$ and the results were shown in Figure 10.

It is clear from Figure 10(a) that there are three congested roads and the congestion spreads upstream gradually. However, the phase plane diagrams mainly described the instability of the system. When the vehicle density becomes saturated, the state variable $\eta$ increases greatly. So we can see three curves changing greatly from Figure 10(b). But most small amplitude traffic flow fluctuations account for a small proportion in the graphs and they are just centered in a small area near the initial value.

\section{Conclusions}

The existing models cannot fully describe the phenomena raised by different input and output on ramps and there are few studies on the traffic phenomena using the phase plane diagrams. In this paper, we present an improved macro model for traffic flow on a highway with ramps based on the existing models. Furthermore, two new variable substitutions are adopted to extend the range of the variable from a specific value to infinity, so the model is transformed into a new model which is suitable for the stability analysis in phase plane. The problem of traffic flow could be converted into that of system stability. When the input flow of the on-ramp is equal to the output flow of the off-ramp, the equilibrium point equation of the model is calculated and the stop-andgo traffic is described in phase plane. The results show that the traffic phenomena described by the new model are consistent with those of real traffic, which shows that the new model is reasonable. When the stop-and-go waves appear and the traffic flow fluctuations tend to be unstable, the curves in phase plane diagrams are divergent and many of them tend to infinity. The phase plane analysis highlights the unstable traffic phenomena we are chiefly concerned about and describes the variation of density or velocity with time or sections more clearly. When the input flow of the on-ramp is unequal to the output flow of the off-ramp, some traffic phenomena on a highway with ramps are found by our model. The numerical simulation shows that the model can reproduce some complex phenomena of fixed vehicle generation rate but increasing initial homogeneous density with a single ramp and the situation of morning peak. According to the actual road sections of Xian-Baoji highways, the traffic phenomena on a highway with multiple ramps are also analyzed. As all these numerical results are consistent with general traffic situations, the new model is reasonable. Moreover, the phase plane diagrams highlight the instability of the system. As long as the traffic becomes congested, the curves will be divergent and approach infinity in phase plane. However, the continuously changing vehicle generation rate and the varying initial density can pose more complex traffic phenomena. Our ongoing research will study various phenomena induced by the ramps, develop a ramp model that takes more factors into consideration, such as the road conditions, and examine the validation of the model using actual traffic data collected from the field.

\section{Conflict of Interests}

The authors declare that there is no conflict of interests regarding the publication of this paper.

\section{Acknowledgments}

The authors would like to thank the anonymous referees and the editor for their valuable opinions. This work is partially supported by the National Nature Science Foundation of China under the Grant no. 61134004.

\section{References}

[1] X. S. Hu, N. Murgovski, L. Johannesson, and B. Egardt, "Energy efficiency analysis of a series plug-in hybrid electric bus with 
different energy management strategies and battery sizes," Applied Energy, vol. 111, pp. 1001-1009, 2013.

[2] X. S. Hu, N. Murgovski, L. M. Johannesson, and B. Egardt, "Comparison of three electrochemical energy buffers applied to a hybrid bus powertrain with simultaneous optimal sizing and energy management," IEEE Transactions on Intelligent Transportation Systems, vol. 15, no. 3, pp. 1193-1205, 2014.

[3] X. S. Hu, N. Murgovski, L. M. Johannesson, and B. Egardt, "Optimal dimensioning and power management of a fuel cell/battery hybrid bus via convex programming," IEEE/ASME Transactions on Mechatronics, vol. 20, no. 1, pp. 457-468, 2015.

[4] C. Sun, S. J. Moura, X. S. Hu, J. K. Hedrick, and F. C. Sun, "Dynamic traffic feedback data enabled energy management in plug-in hybrid electric vehicles," IEEE Transactions on Control Systems Technology, vol. 23, no. 3, pp. 1075-1086, 2015.

[5] C. Sun, X. S. Hu, S. J. Moura, and F. C. Sun, "Velocity predictors for predictive energy management in hybrid electric vehicles," IEEE Transactions on Control Systems Technology, vol. 23, no. 3, pp. 1197-1204, 2015.

[6] H. Y. Lee, H.-W. Lee, and D. Kim, "Origin of synchronized traffic flow on highways and its dynamic phase transitions," Physical Review Letters, vol. 81, no. 5, pp. 1130-1133, 1998.

[7] A. K. Gupta and V. K. Katiyar, "Phase transition of traffic states with on-ramp," Physica A: Statistical Mechanics and Its Applications, vol. 371, no. 2, pp. 674-682, 2006.

[8] D.-W. Huang, "Ramp-induced transitions in traffic dynamics," Physical Review E, vol. 73, no. 1, Article ID 016123, 2006.

[9] T.-Q. Tang, H.-J. Huang, S. C. Wong, Z.-Y. Gao, and Y. Zhang, "A new macro model for traffic flow on a highway with ramps and numerical tests," Communications in Theoretical Physics, vol. 51, no. 1, pp. 71-78, 2009.

[10] M. J. Lighthill and G. B. Whitham, "On kinematic waves: II. A theory of traffic flow on long crowed roads," Proceedings of the Royal Society of London, vol. 229, pp. 317-345, 1955.

[11] P. I. Richards, "Shock waves on the highway," Operations Research, vol. 4, pp. 42-51, 1956.

[12] H. J. Payne, Models of Freeway Traffic and Control, vol. 1 of Simulation Council Proceedings: Mathematical Models of Public Systems, Simulation Councils, La Jolla, Calif, USA, 1971.

[13] W. F. Phillips, "A kinetic model for traffic flow with continuum implications," Transportation Planning and Technology, vol. 5, no. 3, pp. 131-138, 1979.

[14] P. Ross, “Traffic dynamics," Transportation Research. Part B. Methodological, vol. 22, no. 6, pp. 421-435, 1988.

[15] P. G. Michalopoulos, P. Yi, and A. S. Lyrintzis, "Continuum modelling of traffic dynamics for congested freeways," Transportation Research Part B, vol. 27, no. 4, pp. 315-332, 1993.

[16] H. M. Zhang, "Anisotropic property revisited-does it hold in multi-lane traffic?" Transportation Research Part B: Methodological, vol. 37, no. 6, pp. 561-577, 2003.

[17] A. K. Gupta and V. K. Katiyar, "Analyses of shock waves and jams in traffic flow," Journal of Physics A. Mathematical and General, vol. 38, no. 19, pp. 4069-4083, 2005.

[18] B. S. Kerner and P. Konhäuser, "Cluster effect in initially homogeneous traffic flow," Physical Review E, vol. 48, no. 4, pp. 23352338, 1993.

[19] R. Jiang, M.-B. Hu, B. Jia, R. Wang, and Q.-S. Wu, "Spatiotemporal congested traffic patterns in macroscopic version of the Kerner-Klenov speed adaptation model," Physics Letters A, vol. 365 , no. 1-2, pp. 6-9, 2007.
[20] R. Jiang, Q.-S. Wu, and Z.-J. Zhu, "A new continuum model for traffic flow and numerical tests," Transportation Research Part B: Methodological, vol. 36, no. 5, pp. 405-419, 2002.

[21] L. Yu, T. Li, and Z.-K. Shi, "The effect of diffusion in a new viscous continuum traffic model," Physics Letters, Section A: General, Atomic and Solid State Physics, vol. 374, no. 23, pp. 2346-2355, 2010.

[22] H. X. Ge and X. L. Han, "Density viscous continuum traffic flow model," Physica A, vol. 371, no. 2, pp. 667-673, 2006.

[23] A. K. Gupta and S. Sharma, "Analysis of the wave properties of a new two-lane continuum model with the coupling effect," Chinese Physics B, vol. 21, no. 1, Article ID 015201, 2012.

[24] L. Yu and Z.-K. Shi, "Density wave in a new anisotropic continuum model for traffic flow," International Journal of Modern Physics C, vol. 20, no. 11, pp. 1849-1859, 2009. 


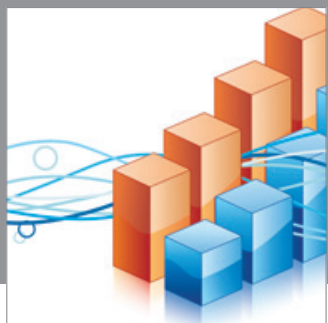

Advances in

Operations Research

mansans

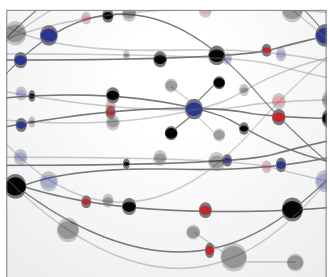

The Scientific World Journal
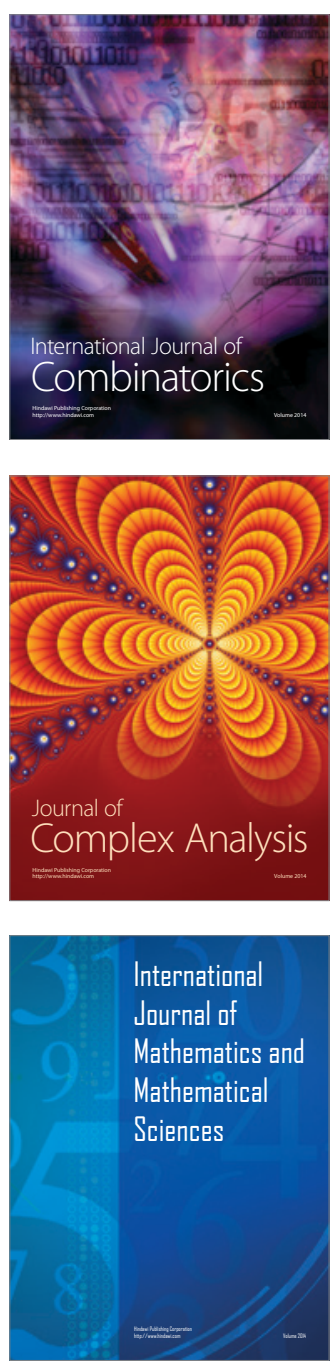
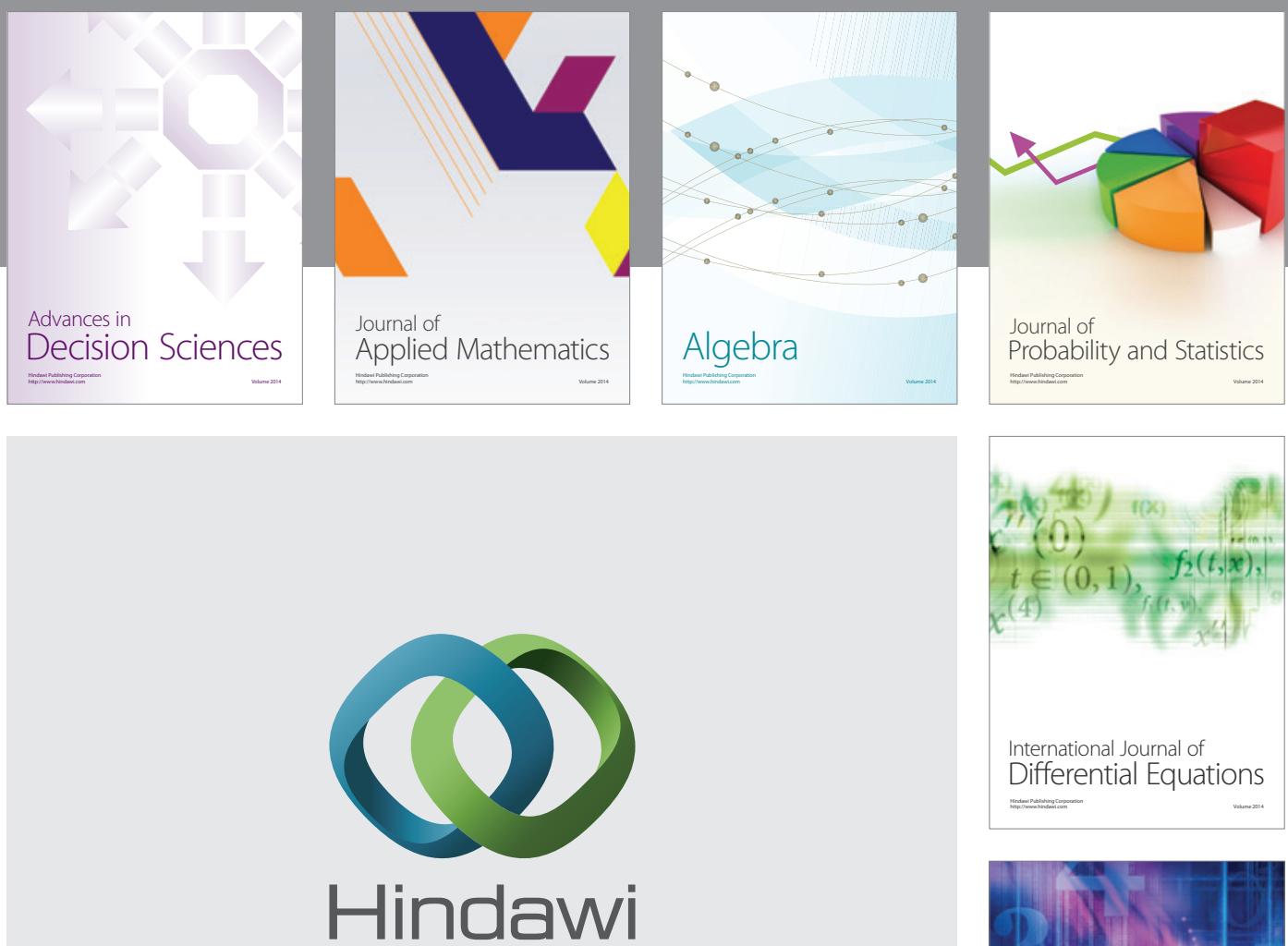

Submit your manuscripts at http://www.hindawi.com
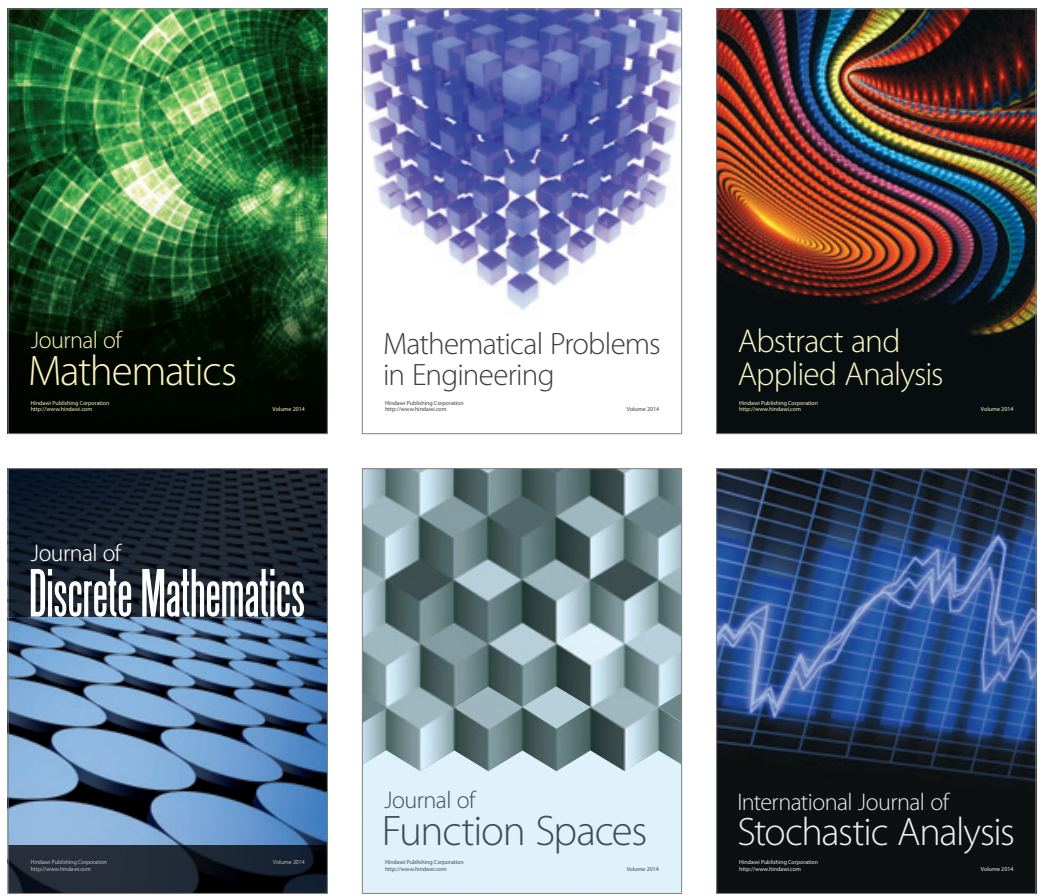

Journal of

Function Spaces

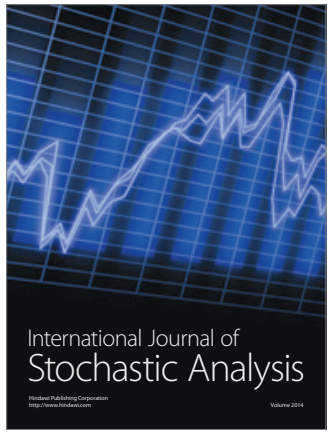

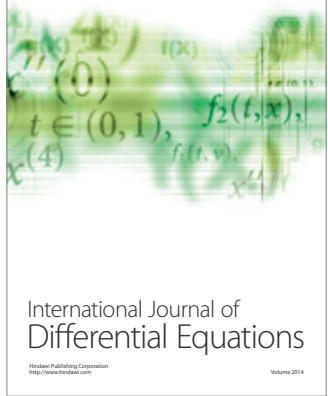
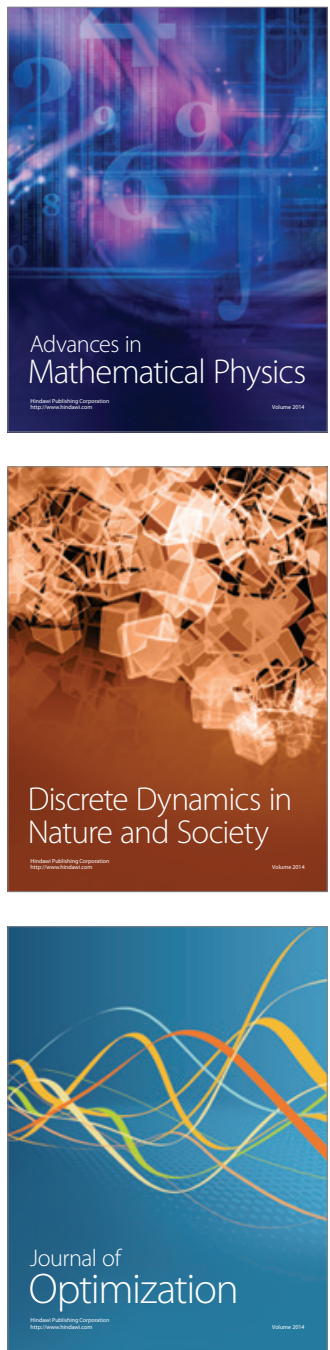